\title{
Weighted Fuzzy Generalized 2DFLD: A Fuzzy-Based Feature Extraction Technique for Face Recognition
}

\author{
Aniruddha Dey, Jamuna Kanta Sing, and Shiladitya Chowdhury
}

\begin{abstract}
This paper proposes a weighted scheme and fuzzy logic-based feature extraction technique, called weighted fuzzy generalized two-dimensional Fisher's linear discriminant (WFG-2DFLD) and its use for face recognition using radial basis function (RBF) neural network as a classifier. In particular, the WFG-2DFLD method is extended version of the generalized two-dimensional Fisher's linear discriminant (G-2DFLD) method. Like G-2DFLD, WFG-2DFLD also maximizes class separability along row and column directions simultaneously. Firstly, it calculates fuzzy membership matrix by fuzzy $k$-nearest neighbour (F $k$-NN) algorithm for the training samples. Secondly, the fuzzy membership values are combined with the training samples to obtained global mean and class-wise mean training images. Thereafter, the global and class-wise mean images are used to generate fuzzy within-class and fuzzy between-class scatter matrices along the row and column directions. In order to make more accurate for classification, different weights are incorporated to scatter matrices. Finally, by solving the Eigen value problems of these scatter matrices; we find the optimal fuzzy projection vectors, which actually used to generate more discriminant features for face recognition. The WFG-2DFLD method has been evaluated on the YALE, AT\&T (formally known as ORL), UMIST and FERET face databases using RBF neural network. Simulation results demonstrate that the proposed WFG-2DFLD method can obtain higher recognition rates than some state-of-the-art face recognition methods.
\end{abstract}

Index Terms-WFG-2DFLD, fuzzy projection vector, $\mathrm{F} k$-NN, RBFNN based classifier, matrix-based feature extraction.

\section{INTRODUCTION}

In the last few decades the pattern recognition, biometrics and feature extraction became very popular research area among the researchers [1]-[4]. In this context, the face recognition problem can be viewed as a classification problem where one or more test images are compared with the face images stored in the databases. Although the face recognition techniques from still images having controlled background have matured substantially, the face recognition task is still very challenging under uncontrolled environment and different issues related to human face i.e. facial expression (unhappiness, happiness) and facial pose, occlusion (wearing beards, mustaches and glasses), illumination variance, etc. Broadly, the face recognition

Manuscript received August 21, 2017; revised November 10, 2017.

Aniruddha Dey and Jamuna Kanta Sing are with the Department of Computer Science Engineering, Jadavpur University, Kolkata, India (e-mail: anidey007@gmail.com,jksing@ieee.org).

Shiladitya Chowdhury is with the Department of Computer Application, Techno India, Kolkata, India (e-mail: dityashila@yahoo.com). methods can be classified into three main categories: $(a)$ holistic approach, (b) feature based approach and (c) hybrid approach [1]-[9].

Holistic approach: This approach uses all the information available in the face images as a whole. The subspace-based methods, like principal component analysis (PCA), linear discriminant analysis (LDA) or Fisher's linear discriminant (FLD) [2], [3] and their variants, which use eigenfaces and/or fisherface to compute features, fall under this category. In particular, PCA maximizes the total scatter matrix across all face images. However, undesirable variations caused by lighting, facial expression and other factors are retained through PCA techniques. Many researchers argue that the PCA techniques do not provide any information for class discrimination; only perform dimension reduction [3]. The LDA has been proposed as a better alternative to the PCA to provide class discrimination information [3], [4]. The main objective of the LDA is to find best discrimination of vectors among the classes by maximizing the between-class differences and minimizing the within-class ones [3]. The main shortcoming of the LDA technique in face recognition is the "small sample size (SSS)" problem [4]. The SSS problem generally arises when the number of samples (images) is smaller than the dimension of the samples. The dimension of face images is generally very high; as a results, the within-class scatter matrix become singular that makes the FLD method infeasible. The SSS problem in LDA can be solved by sampling down the face images into smaller size. To avoid SSS problem PCA + FLD technique was proposed by Er. et al. [5]. They have used the PCA method to reduce dimension of face images and then used the FLD technique to obtain lower dimensional discriminant features [5], [6]. Independent component analysis (ICA) is also developed as an effective feature extraction technique [6]. ICA computes discriminant features from covariance matrix by considering high-order statistics. The two-dimensional PCA (2DPCA) directly works on the $2 \mathrm{D}$ image matrices and found to be computationally efficient and more superior for face recognition and reconstruction than PCA [7]. Two-dimensional FLD (2DFLD) method also works directly on the 2D image matrices and maximizes class separability either from row or column direction [8]. The 2DFLD method is much superior in comparison to PCA and 2DPCA methods in terms of feature extraction and face recognition [9]. An improvement of the 2DFLD method, the generalized 2DFLD (G-2DFLD) method brings out the projection vectors both from the row and column directions from the training images [9]. Then, linearly projecting an image matrix on these row and column projection vectors the discriminant feature vectors are obtained in the form of a matrix. Consequently, this algorithm also maximizes the discriminative information 
among the classes while minimizing it within a class [9].

Recently, researchers have devised different fuzzy-based techniques $e . g$. fuzzy fisherface, fuzzy $k$-nearest neighbour $(\mathrm{F} k-\mathrm{NN})$, fuzzy two dimensional principal component analysis (F-2DPCA) and fuzzy fisherface, fuzzy two dimensional Fisher's linear discrimination (F-2DFLD). The aforementioned techniques are mainly used in facial feature extraction and face recognition [10]-[15]. Authors in [10] have devised fuzzy $k$-nearest neighbour ( $k k-N N)$ approach, where the fuzzification is done on the class assignment. The fuzzy fisherface (Fuzzy-FLD) method is an extension of fisherface method where fuzzy logic is combined with the face recognition technique [11]. The Fuzzy-FLD method defines the within- and between-class scatter matrices for binary labeled patterns by incorporating the fuzzy membership grades. These matrices are used for face recognition [11]. Again, the fuzzy fisherface is extended to the fuzzy 2DFLD (F-2DFLD) where the scatter matrices were redefined by integrating the membership grades into each training sample [12]. Yang et al. proposed feature extraction using fuzzy inverse FDA [13]. The membership degree matrices are calculated in this context by incorporating the Fk-NN. The Fuzzy 2DPCA technique is presented where $\mathrm{F} k$-NN method is also used to calculate the membership matrix for training sample [14]. The maximum scatter difference (MSD) is further extended to fuzzy MSD [15].

\section{A. Feature Based Approach}

Feature based approach uses facial structural information for feature extraction and recognition [18], [19]. However, all of these techniques mainly depend on heuristics search subspace with geometrical constraints. The elastic bunch graph matching method, where face recognition is formulated by elastic graph matching that is executed by stochastic optimization of a matching cost function with an additional computational overhead [20], [21]. The hidden Markov model (HMM)-based methods use strip of pixels to cover eye, nose, mouth, forehead and chin without locating the exact view of the facial features [20]. The dynamic link architecture (DLA)-based graph matching technique is one of the most popular methods in the categories [21].

\section{B. Hybrid Approach}

This approach uses both local features and the whole face image for feature extraction and recognition [22]-[25]. It is more similar to the behavioral aspect of human being to recognize a face. These types of methods try to realize the human perception by integrating holistic and feature-based approaches to recognize a face. Some of the hybrid methods are the modular eigenfaces method [22], hybrid local feature analysis (LFA) [23], shape normalized method [24] and component-based method [25]. The modular eigenfaces method [22] uses hybrid features by combining eigenfaces and other Eigen modules i.e. eyes, mouth and nose. This method is found to be slightly superior to the holistic eigenfaces method. The hybrid LFA method [23] uses a set of hybrid features using PCA and LFA methods. The shape normalized method uses both shape and gray-level information for automatic face recognition [24]. The component-based method [25] decomposes a face into a set of facial components such as mouth and eyes that are interconnected by a flexible geometrical model. One drawback of this method is that it needs a large number of training images taken from different viewpoints and under different lighting conditions.

In the proposed method, we have incorporated the fuzzy membership values in different classes which are computed from the training images (samples). To obtain the membership degrees of each training sample, we have used the fuzzy $k$-NN and used them for calculating the global and class-wise mean training image matrices. Finally, the fuzzy between- and within-class scatter matrices are calculated separately in row and column directions. The features are extracted by solving the eigenvalue problem of these scatter matrices.

The paper is organized as follows: related feature extraction methods are described in Section II. The outline of $\mathrm{F} k$-NN, brief description, algorithm and implementation details of proposed WFG-2DFLD method is elaborated in Section III. The key idea of RBFNN is represented in Section IV. The empirical results on four public face image datasets are illustrated in Section V. Finally, a discussion in Section VI draws the concluding remarks of the paper.

\section{RELATED FEATURE EXTRACTION TECHNIQUES}

Our proposed method is based on the generalized two-dimensional Fisher's linear discriminant (G-2DFLD) [9] and weighted fuzzy maximum scatter difference (FMSD) [15] feature extraction techniques. To make paper self-contained and also to distinguish our contribution, the G-2DFLD and FMSD methods are briefly presented in this section.

\section{A. Overview of the Generalized 2DFLD Method for Feature Extraction}

Let, the face images are of $r \times s$ dimension which are represented in the form of $2 \mathrm{D}$ vectors $\boldsymbol{X}_{i}(i=1,2, \ldots, N)$. The total number of " $C$ " classes comprises $N$ face images. The $c^{\text {th }}$ class is represented by $C_{c}$ having total samples of $N_{c}$ and also satisfying the condition $\left(\sum_{c=1}^{C} N_{c}=N\right)$. The global mean training images denoted by $\boldsymbol{v}=\frac{1}{N} \sum_{i=1}^{N} \boldsymbol{X}_{i}$, and class-wise mean training images denoted by $\boldsymbol{v}_{c}=$ $\frac{1}{N_{c}} \sum_{i=1}^{N} \boldsymbol{X}_{i} \mid \boldsymbol{X}_{i} \in C_{c}$. Given an image $\boldsymbol{X}$, the G-2DFLD -based [9] 2D feature matrix $\boldsymbol{Y}$ is generated by the following linear transformation:

$$
\boldsymbol{Y}=\left(\boldsymbol{P}_{\text {opt }}\right)^{T} \boldsymbol{X}\left(\boldsymbol{Q}_{\text {opt }}\right)
$$

where $\boldsymbol{P}_{\text {opt }}$ and $\boldsymbol{Q}_{\text {opt }}$ are the two optimal projection matrices.

The between-class and within-class scatter matrices along row direction ( $\boldsymbol{S}_{r b}$ and $\left.\boldsymbol{S}_{r w}\right)$ and column direction $\left(\boldsymbol{S}_{c b}\right.$ and $\left.\boldsymbol{S}_{c w}\right)$ are computed as follows:

$$
\begin{gathered}
\boldsymbol{S}_{r b}=\sum_{c=1}^{C} N_{c}\left(\boldsymbol{v}_{c}-\boldsymbol{v}\right)\left(\boldsymbol{v}_{c}-\boldsymbol{v}\right)^{T} \\
\boldsymbol{S}_{r w}=\sum_{c=1}^{C} \sum_{i \in c}^{N}\left(\boldsymbol{X}_{i}-\boldsymbol{v}_{c}\right)\left(\boldsymbol{X}_{i}-\boldsymbol{v}_{c}\right)^{T} \\
\boldsymbol{S}_{c b}=\sum_{c=1}^{C} N_{c}\left(\boldsymbol{v}_{c}-\boldsymbol{v}\right)^{T}\left(\boldsymbol{v}_{c}-\boldsymbol{v}\right) \\
\boldsymbol{S}_{c w}=\sum_{c=1}^{C} \sum_{i \in c}^{N}\left(\boldsymbol{X}_{i}-\boldsymbol{v}_{c}\right)^{T}\left(\boldsymbol{X}_{i}-\boldsymbol{v}_{c}\right)
\end{gathered}
$$

In above expression, the dimensions of the row-wise scatter matrices $\left(\boldsymbol{S}_{r b}\right.$ and $\left.\boldsymbol{S}_{r w}\right)$ and the column-wise scatter matrices $\left(\boldsymbol{S}_{c b}\right.$ and $\left.\boldsymbol{S}_{c w}\right)$ are found to be $r \times r$ and $s \times s$, respectively. 
The two Fisher's criteria (objective function) along row direction $(J(\boldsymbol{P}))$ and column direction $(J(\boldsymbol{Q}))$ have been formulated as stated below:

$$
\begin{aligned}
& J(\boldsymbol{P})=\frac{\left|\boldsymbol{P}^{T} \boldsymbol{S}_{r b} \boldsymbol{P}\right|}{\left|\boldsymbol{P}^{T} \boldsymbol{S}_{r w} \boldsymbol{P}\right|} \\
& J(\boldsymbol{Q})=\frac{\left|\boldsymbol{Q}^{T} \boldsymbol{S}_{c b} \boldsymbol{Q}\right|}{\left|\boldsymbol{Q}^{T} \boldsymbol{S}_{c w} \boldsymbol{Q}\right|}
\end{aligned}
$$

The optimal projection vectors $\boldsymbol{P}_{\text {opt }}$ and $\boldsymbol{Q}_{\text {opt }}$ can be obtained by finding the normalized eigenvalues the eigenvectors of $\boldsymbol{S}_{r b} \boldsymbol{S}_{r w}^{-1}$ and $\boldsymbol{S}_{c b} \boldsymbol{S}_{c w}^{-1}$, respectively. The eigenvalues are sorted in descending order and the eigenvectors are also rearranged accordingly. The optimal projection (eigenvector) matrix $\boldsymbol{P}_{\text {opt }}$ and $\boldsymbol{Q}_{\text {opt }}$ can be formed as follows:

$$
\begin{aligned}
\boldsymbol{P}_{o p t} & =\arg \max _{P}\left|\boldsymbol{S}_{r b} \boldsymbol{S}_{r w}^{-1}\right| \\
& =\left[p_{1}, p_{2}, \ldots, p_{u}\right] \\
\boldsymbol{Q}_{o p t} & =\arg \max _{Q}\left|\boldsymbol{S}_{c b} \boldsymbol{S}_{c w}^{-1}\right| \\
& =\left[q_{1}, q_{2}, \ldots, q_{v}\right]
\end{aligned}
$$

B. Overview of the Weighted FMSD Method for Feature Extraction

In the FMSD method [15] the fuzzy membership value is incorporated into the within and between scatter matrices.

Step 1: $(\mathrm{F} k-\mathrm{NN})$ : The scatter matrices are then redefined by integrating the fuzzy membership $\left(M_{F}\right)$ with the training images [10].

Step 2: Calculate the global mean training image $(\overline{\boldsymbol{\tau}}=$ $\left.\frac{\sum_{c=1}^{C} \sum_{i=1}^{N} \boldsymbol{\mu}_{c i} \boldsymbol{X}_{i}}{\sum_{c=1}^{C} \sum_{i=1}^{N} \boldsymbol{\mu}_{c i}}\right)$ and class-wise mean training image $\left(\overline{\boldsymbol{\tau}}_{c}=\right.$ $\left.\frac{\sum_{i=1}^{N} \boldsymbol{\mu}_{c i} \boldsymbol{X}_{i}}{\sum_{i=1}^{N} \boldsymbol{\mu}_{c i}} ; c=1,2,3, \ldots, C\right)$. The fuzzy membership degrees are incorporated into the training images to obtain the global- and class-wise mean images [15].

Step 3: The fuzzy between image scatter $\left(\boldsymbol{S}_{b}^{f}\right)$ and fuzzy within image scatter matrices $\left(\boldsymbol{S}_{w}^{f}\right)$ and Fisher's criteria (objective function) $J\left(\boldsymbol{W}^{f}\right)$ are formulated as follows:

$$
\left\{\begin{array}{l}
\boldsymbol{S}_{b}^{f}=\frac{1}{N} \sum_{c=1}^{C} N_{c}\left(\overline{\boldsymbol{\tau}}_{c}-\overline{\boldsymbol{\tau}}\right)\left(\overline{\boldsymbol{\tau}}_{c}-\overline{\boldsymbol{\tau}}\right)^{T} \\
\boldsymbol{S}_{w}^{f}=\frac{1}{N} \sum_{c=1}^{C} \sum_{i \in c}^{N}\left(X_{i}-\overline{\boldsymbol{\tau}}_{c}\right)^{T}\left(X_{i}-\overline{\boldsymbol{\tau}}_{c}\right) \\
J\left(\boldsymbol{W}^{f}\right)=\arg \max _{\boldsymbol{W}^{f}} \frac{\left|\left(\boldsymbol{W}^{f}\right)^{T} \boldsymbol{S}_{\boldsymbol{b}}^{f} \boldsymbol{W}^{f} \beta\right|}{\left|\left(\boldsymbol{W}^{f}\right)^{T} \boldsymbol{S}_{\boldsymbol{w}}^{f} \boldsymbol{W}^{f}(1-\beta)\right|}
\end{array}\right.
$$

where $0<\beta<1$

where the total number of " $C$ " classes comprises $N$ face images. The $c^{\text {th }}$ class is represented by $C_{c}$ having total samples of $N_{c}$ and also satisfying the condition $\left(\sum_{c=1}^{C} N_{c}=\right.$ $N)$.

Step 4: Project all samples to find the optimal projection vectors $\boldsymbol{W}_{\text {opt }}^{f}$ and classify testing samples with the classifier.

\section{FrAMEWORK (AlgORITHM) OF PROPOSED WEIGHTED FUZZY GENERALIZED TWO-DIMENSIONAL LINEAR DISCRIMINANT (WFG-2DFLD) METHOD}

The human face images are different under various circumstances such as, illumination, pose etc. As a result, sometimes, images of a person may look alike to that of a different person. In addition, variability among the images of a person may rise quite significantly. Therefore, fuzzy class assignment for the training images may be suitable instead of binary class assignment. In this study, to minimize the distance between the within-class samples and to maximize the between-class samples, the weights are incorporated. This enables more robust and easy classification of the images. The discriminant features are extracted by setting the weighted parameter $\beta$ as it gives optimal result in this study. The proposed WFG-2DFLD method is basically based on the concept of fuzzy class assignment, where a face image belongs to different classes as characterized by its fuzzy membership values. In the present study, we have used the F $k$-NN [10] for generating fuzzy membership values for training images; resulting a fuzzy membership matrix. The fuzzy membership values are incorporated with the training images to obtain global and class-wise mean images, which in turn used to form fuzzy between-class and fuzzy within-class scatter matrices. Therefore, these scatter matrices yield useful information regarding association of each training image into several classes. The optimal fuzzy $2 \mathrm{D}$ projection vectors are obtained by solving the eigenvalue problems of these scatter matrices. Finally, the WFG-2DFLD -based features are extracted by projecting a face image onto these optimal fuzzy $2 \mathrm{D}$ projection vectors. The different steps of the WFG-2DFLD method are presented in details in the following sub-sections.

\section{A. Generation of Membership Matrix by Fuzzy k-Nearest Neighbor $(F k-N N)$}

Let, there are $C$ classes and $N$ training images; each one is represented in the form of $2 \mathrm{D}$ vectors $\boldsymbol{X}_{i}(i=1,2, \ldots, N)$. A fuzzy $k$-NN-based decision method has been used for assigning membership values to the training samples (images). The steps for computations of the membership degrees are described as follows [10, 11]:

Step 1: Euclidean distance matrix is calculated between the pair of training images. Row and column indexes represent the serial number of the training images. In addition, generate a 2D class matrix, whose column entries represent the class values corresponding to the training images.

Step 2: Diagonal values (usually ' 0 ') of this matrix are replaced by large numeric values, which is greater than the maximum value of its own column.

Step 3: Sort (ascending order) the distance matrix in a column-wise direction and the corresponding class matrix is also rearranged accordingly.

Step 4: The class labels are collected from the patterns in column-wise direction, located in the nearest neighborhood according to the $k$-nearest neighbors rule and a list of " $k$ " integers are returned.

Step 5: Compute membership degree value $\mu_{i j}$ for the $j^{\text {th }}$ image that belongs to $i^{\text {th }}$ class according to the following equation:

$$
\mu_{i j}=\left\{\begin{array}{rr}
0.51+0.49\left(\frac{n_{i j}}{k}\right) ; \text { if } i \text { is equal to the } \\
\text { class of the } j^{t h} \text { image } \\
0.49\left(\frac{n_{i j}}{k}\right) ; & \text { otherwise }
\end{array}\right.
$$


where $n_{i j}$ represents the total number of neighbors of the $j^{t h}$ image which belong to the $i^{\text {th }}$ class.

The method actually redefines the membership values of the labelled images. When, all of the neighbors belong to the $i^{\text {th }}$ class which is equal to the class of $j^{\text {th }}$ image under consideration, then $n_{i j}=k$ and $\mu_{i j}$ returns 1 , making membership values for the other classes as zero. In addition, $\mu_{i j}$ also satisfies two properties as stated below:

$$
\left.\begin{array}{l}
\sum_{i=1}^{C} \mu_{i j}=1 \\
\text { and } \\
0<\sum_{j=1}^{N} \mu_{i j}<N
\end{array}\right\}
$$

So, the fuzzy membership matrix $M_{F}$ using the F $k$-NN can be illustrated as given below:

$$
M_{F}=\left[\mu_{c i}\right] ; c=1,2,3, \ldots, C ; \quad i=1,2,3, \ldots, N
$$

\section{B. Generation of Optimal Weighted Fuzzy Projection Vectors}

The next step of the proposed WFG-2DFLD method is to integrate the fuzzy membership values with the training images and redefine the scatter matrices along row and column directions. The discriminant features are extracted by setting the weighted parameter. Finally, generate the optimal fuzzy projection vectors by solving the eigenvalue problems of these scatter matrices.

Let the training set contains $N$ images of $C$ classes (subjects) and each one is denoted as $\boldsymbol{X}_{i}(i=1,2,3 \ldots, N)$ having dimension as $r \times s$. The $c^{\text {th }}$ class $C_{c}$, has total $N_{c}$ images and satisfies $\sum_{c=1}^{C} N_{c}=N$. Let $\overline{\boldsymbol{\tau}}$ is the global mean image and $\overline{\boldsymbol{\tau}}_{c}$ is the mean image of the $c^{\text {th }}$ class. Fuzzy membership degrees are incorporated into the training images to get global mean image and class-wise mean images, which are defined as follows:

$$
\begin{gathered}
\overline{\boldsymbol{\tau}}_{c}=\frac{\sum_{i=1}^{N} \boldsymbol{\mu}_{c i} \boldsymbol{X}_{i}}{\sum_{i=1}^{N} \boldsymbol{\mu}_{c i}} ; c=1,2,3, \ldots, C \\
\overline{\boldsymbol{\tau}}=\frac{\sum_{c=1}^{C} \sum_{i=1}^{N} \boldsymbol{\mu}_{c i} \boldsymbol{X}_{i}}{\sum_{c=1}^{C} \sum_{i=1}^{N} \boldsymbol{\mu}_{c i}}
\end{gathered}
$$

The four scatter matrices along the row and column directions are redefined as (i) fuzzy image row-wise between-class scatter matrix, (ii) fuzzy image row-wise within-class scatter matrix, (iii) fuzzy column-wise between-class scatter matrix and (iv) fuzzy column-wise within-class scatter matrix and denoted as $\boldsymbol{S}_{r b}^{f}, \boldsymbol{S}_{r w}^{f}, \boldsymbol{S}_{c b}^{f}$, $\boldsymbol{S}_{c w}^{f}$, respectively. These fuzzy scatter matrices $\left(\boldsymbol{S}_{r b}^{f}, \boldsymbol{S}_{r w}^{f}\right.$, $\left.\boldsymbol{S}_{c b}^{f}, \boldsymbol{S}_{c w}^{f}\right)$ are expressed as follows:

$$
\begin{gathered}
\boldsymbol{S}_{r b}^{f}=\frac{1}{N} \sum_{c=1}^{C} N_{c}\left(\overline{\boldsymbol{\tau}}_{c}-\overline{\boldsymbol{\tau}}\right)\left(\overline{\boldsymbol{\tau}}_{c}-\overline{\boldsymbol{\tau}}\right)^{T} \\
\boldsymbol{S}_{r w}^{f}=\frac{1}{N} \sum_{c=1}^{C} \sum_{i \in c}^{N}\left(\boldsymbol{X}_{i}-\overline{\boldsymbol{\tau}}_{c}\right)\left(\boldsymbol{X}_{i}-\overline{\boldsymbol{\tau}}_{c}\right)^{T} \\
\boldsymbol{S}_{c b}^{f}=\frac{1}{N} \sum_{c=1}^{C} N_{c}\left(\overline{\boldsymbol{\tau}}_{c}-\overline{\boldsymbol{\tau}}\right)^{T}\left(\overline{\boldsymbol{\tau}}_{c}-\overline{\boldsymbol{\tau}}\right) \\
\boldsymbol{S}_{c w}^{f}=\frac{1}{N} \sum_{c=1}^{C} \sum_{i \in c}^{N}\left(\boldsymbol{X}_{i}-\overline{\boldsymbol{\tau}}_{c}\right)^{T}\left(\boldsymbol{X}_{i}-\overline{\boldsymbol{\tau}}_{c}\right)
\end{gathered}
$$

It may be noted that the size of the $\boldsymbol{S}_{r b}^{f}$ and $\boldsymbol{S}_{r w}^{f}$ scatter matrices is $r \times r$; whereas for the $\boldsymbol{S}_{c b}^{f}$ and $\boldsymbol{S}_{c w}^{f}$ scatter matrices it is $s \times s$.
The Fisher's criteria (objective function) $J_{w}^{f}\left(\boldsymbol{W}^{f^{\prime}}\right)$ and $J_{w}^{f}\left(\boldsymbol{W}^{f^{\prime \prime}}\right)$ along row and column directions are defined as follows:

$$
J_{w}^{f}\left(\boldsymbol{W}^{f^{\prime}}\right)=\arg \max _{\boldsymbol{W} f^{\prime}} \frac{\left|\left(\boldsymbol{W}^{f^{\prime}}\right)^{T} \boldsymbol{s}_{r b}^{f} \boldsymbol{W}^{f^{\prime}} \beta\right|}{\left|\left(\boldsymbol{W}^{f^{\prime}}\right)^{T} \boldsymbol{S}_{r w}^{f} \boldsymbol{W}^{f^{\prime}}(1-\beta)\right|}
$$

and

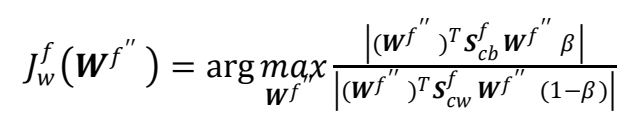

where $0<\beta<1$

The ratio is maximized in the above two equations (20) and (21) when the row and the column vectors of the weighted fuzzy projection matrix $\boldsymbol{W}^{f^{\prime}}$ and $\boldsymbol{W}^{f^{\prime \prime}}$ are the eigenvectors of $\boldsymbol{S}_{r b}^{f} \beta\left(\boldsymbol{S}_{r w}^{f}(1-\beta)\right)^{-1} \quad$ and $\boldsymbol{S}_{c b}^{f} \beta\left(\boldsymbol{S}_{c w}^{f}(1-\beta)\right)^{-1}$, respectively. The weighted fuzzy optimal projection matrix $\boldsymbol{W}_{o p t}^{f^{\prime}}$ and $\boldsymbol{W}_{o p t}^{f^{\prime \prime}}$ are obtained by finding the eigenvectors of $\boldsymbol{S}_{r b}^{f} \beta\left(\boldsymbol{S}_{r w}^{f}(1-\beta)\right)^{-1} \quad$ and $\quad \boldsymbol{S}_{c b}^{f} \beta\left(\boldsymbol{S}_{c w}^{f}(1-\beta)\right)^{-1}$ corresponding to the $u$ and $v$ largest eigenvalues, respectively. The weighted fuzzy optimal projection matrices $\boldsymbol{W}_{o p t}^{f^{\prime}}$ and $\boldsymbol{W}_{o p t}^{f^{\prime \prime}}$ can be represented as follows:

$$
\begin{aligned}
\boldsymbol{W}_{\text {opt }}^{f^{\prime}} & =\underset{W^{f}}{\arg \max _{\text {ax }}}\left|\boldsymbol{S}_{\text {rb }}^{f} \beta\left(\boldsymbol{S}_{r w}^{f}(1-\beta)\right)^{-1}\right| \\
& =\left[W_{1}^{f^{\prime}}, W_{2}^{f^{\prime}}, \ldots, W_{u}^{f^{\prime}}\right] \\
\boldsymbol{W}_{\text {opt }}^{f^{\prime \prime}} & =\underset{W^{\prime \prime}}{\arg \max _{1}}\left|\boldsymbol{S}_{c b}^{f} \beta\left(\boldsymbol{S}_{c w}^{f}(1-\beta)\right)^{-1}\right| \\
& =\left[W_{1}^{f^{\prime \prime}}, W_{2}^{f^{\prime \prime}}, \ldots, W_{v}^{f^{\prime \prime}}\right]
\end{aligned}
$$

where $\left\{p_{i} \mid i=1,2, \ldots, u\right\}$ is the set of normalized eigenvectors of $\boldsymbol{S}_{r b}^{f} \beta\left(\boldsymbol{S}_{r w}^{f}(1-\beta)\right)^{-1}$ corresponding to $u$ largest eigenvalues $\left\{\lambda_{i} \mid i=1,2, \ldots, u\right\}$ and $\left\{q_{j} \mid j=\right.$ $1,2, \ldots, v\}$ is the set of normalized eigenvectors of $\boldsymbol{S}_{c b}^{f} \beta\left(\boldsymbol{S}_{c w}^{f}(1-\beta)\right)^{-1} \quad$ corresponding to $v$ largest eigenvalues $\left\{\alpha_{j} \mid j=1,2, \ldots, v\right\}$.

\section{Generation of WFG-2DFLD-based Features}

For an image $\boldsymbol{X}$, the WFG-2DFLD-based features in the form of $2 \mathrm{D}$ matrix of size $u \times v$ is generated by projecting it onto the optimal weighted fuzzy projection matrices and can be achieved by the following linear transformation as defined below:

$$
\boldsymbol{Y}_{w}^{f}=\left(\boldsymbol{W}_{o p t}^{f^{\prime}}\right)^{T} \boldsymbol{X}\left(\boldsymbol{W}_{o p t}^{f^{\prime \prime}}\right)
$$

\section{Implementation Details of WEIGHTED Fuzzy G-2DFLD Method}

Step1. (F $\boldsymbol{k}-\mathbf{N N})$ : The matrix of the fuzzy membership degrees $M_{F}$ of the training samples can be calculated by aforesaid $\mathrm{F} k$-NN algorithm. The matrix of the mean image and class-wise mean images are computed according to the fuzzy membership values.

Step2. (WFG-2DFLD): Calculate fuzzy scatter matrices (within-class and between-class) in row- and column-wise directions. Weights $(\beta)$ are multiplied into the above mention scatter matrices. Two objectives functions $J_{w}^{f}\left(\boldsymbol{W}^{f^{\prime}}\right)$ and $J_{w}^{f}\left(\boldsymbol{W}^{f^{\prime \prime}}\right)$ are computed by finding normalized 
eigenvalues and corresponding eigenvectors. Finally, the optimal projection matrix $\boldsymbol{W}^{f^{\prime}}$ and $\boldsymbol{W}^{f^{\prime \prime}}$ are obtained after properly sorting the eigenvectors and are used for feature extraction.

Step3. (Classification): classification and recognition is done after feature extraction using a multilayer RBFNN as classifier.

\section{RADIAL BASIS FUNCTION NEURAL NETWORK}

Once the features are generated by the proposed WFG-2DFLD method for all the images present in the training and test sets, a multilayer radial basis function neural network (RBFNN) is designed and trained using the features of the training images. Thereafter, a test image is classified by the multilayer RBFNN [16, 17] using its WFG-2DFLD -based features. The input layer of the above RBFNN comprises of $D$ units (neurons) for a $D$-dimensional feature (input) vector. Every input unit is fully connected with $L$-hidden layer units excluding the bias unit. The numbers of hidden layer neurons are determined empirically. Likewise, all hidden layer neurons including the bias unit are fully connected with the $C$ units of the output layer which represents the number of classes of the problem. Each hidden layer unit is excited by a Gaussian kernel function and is characterized by its center and the width of the receptive field If the radial distance from the center is zero, the function maximizes and decreases as the distance from the center increases. The Euclidean distance is considered as the measure of distance is expressed as follows:

$$
d\left(\boldsymbol{x}_{i}, \boldsymbol{c}_{j}\right)=\left\|\boldsymbol{x}_{i}-\boldsymbol{c}_{j}\right\|=\left(\sum_{u=1}^{D}\left|x_{i u}-c_{j u}\right|^{2}\right)^{\frac{1}{2}}
$$

where $\boldsymbol{x}_{i}$ is the $i^{\text {th }}$ feature (input) vector, $\boldsymbol{c}_{j}$ is the center of the $j^{\text {th }}$ hidden layer unit and $D$ is the dimension of the input vector.

The output of the $j^{t h}$ hidden layer unit is generated by the following Gaussian function:

$$
\varphi_{j}\left(x_{i}\right)=\exp \left(-\frac{d\left(\boldsymbol{x}_{i}, \boldsymbol{c}_{j}\right)^{2}}{2 \sigma_{j}^{2}}\right)
$$

where $\sigma_{j}$ is the width of the receptive field of the Gaussian function associated with the $j^{\text {th }}$ hidden layer unit. The output of the RBF neural network for $i^{\text {th }}$ input vector $\boldsymbol{x}_{i}$ can be described as below:

$$
z_{i k}=\sum_{j=0}^{L}\left[\varphi_{j}\left(\boldsymbol{x}_{i}\right) w_{k j}\right], \quad k=1,2, \ldots, C
$$

where $w_{k j}$ is the weight between the $j^{\text {th }}$ hidden layer unit and the $k^{t h}$ neuron of the output layer, $\varphi_{0}\left(\boldsymbol{x}_{i}\right)$ and $w_{k 0}$ positive bias having value unity and weight to the $k^{\text {th }}$ output neuron from the bias neuron, respectively. $C$ is the total number of output units of the RBFNN.

The training procedure of the RBF neural network consists of a hybrid process, which passes through two steps one after another. In the first step, the centres of the hidden layer units $\left(\boldsymbol{c}_{j}, j=1,2, \ldots, L\right)$ and the widths $\left(\sigma_{j}\right)$ of the corresponding kernel functions are estimated using an algorithm as described in [16]. While, in the second step, the weights $\left(w_{k j}, k=0,1, \ldots, C\right)$ between the hidden layer and output layer are estimated using the LMS algorithm [16, 17]. The class of a test feature vector (and thus test image) is estimated as the index of the output layer unit, which produces maximum value and is defined as follows:

$$
\operatorname{Class}\left(\boldsymbol{X}_{i}\right)=\arg \max \left\{z_{i k}\right\} ; \quad k=1 \text { to } C
$$

\section{EXPERIMENTAL RESULTS AND DISCUSSION}

In this section, we have assessed the proposed WFG-2DFLD method for facial feature extraction and recognition using four public face databases namely, YALE [27], AT\&T [28], UMIST [29] and FERET [30, 31]. The discriminant features are extracted by setting the weighted parameter $\beta$ as 0.4 as it gives optimal result. The YALE database is used to assess the WFG-2DFLD method under various facial expressions and lighting conditions. The AT\&T database is used to evaluate the presented method under the condition of minor variations of rotation and scaling. The UMIST database is used to evaluate the suggested method when the angle of rotation of the facial images is quite large. Finally, the FERET database is used to evaluate the above proposed method under the condition of variation of facial expression, pose and illumination. In these experiments, we have used a RBFNN classifier due to its superiority and simplicity over the other types of neural networks.

\section{A. YALE Database}

The YALE face database [27] contains face images of 15 different individuals (persons). Each person has 11 images, which are taken under different facial expressions and lighting conditions. Therefore, the YALE database consists of total 165 images. The resolution of each image is of size $320 \times 243$. However, to reduce computational complexity, we have cropped each image into a size of $50 \times 40$ based on the location of eyes. Fig. 1 shows the cropped images of two different individuals.

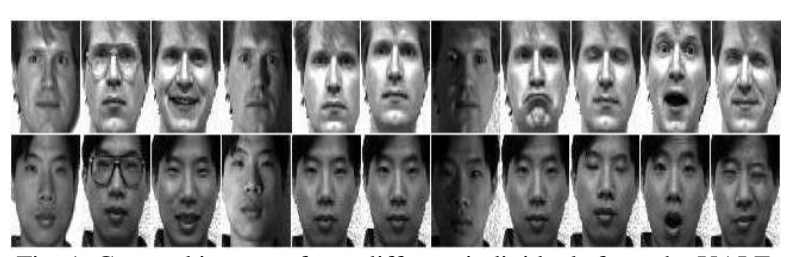

Fig. 1. Cropped images of two different individuals from the YALE database.

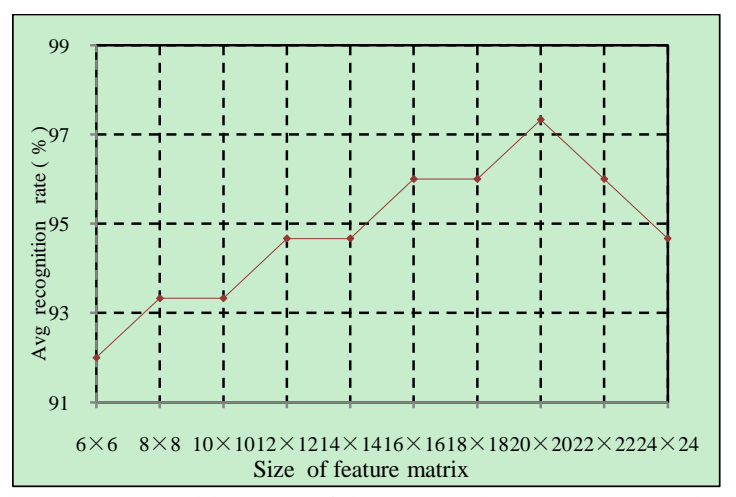

Fig. 2. Average recognition rates of the proposed WFG-2DFLD method on the YALE database by varying the feature matrices. 
For each person, we have taken first six images (i.e. centre-light, with glasses, happy, left-light, without glasses, and normal) for training and rest five of the images (i.e. right-light, sad, sleepy, surprised, and winking) for testing purpose. The WFG-2DFLD method is evaluated with feature matrices of sizes $6 \times 6,8 \times 8,10 \times 10,12 \times 12,14 \times 14,16 \times 16$, $18 \times 18,20 \times 20,22 \times 22$ and $24 \times 24$ using $\mathrm{RBFNN}$ as a classifier. The RBFNN is modeled with 75 hidden layer nodes. Fig. 2 shows performance of the average recognition rates by varying the feature size.

The WFG-2DFLD algorithm achieves $97.33 \%$ recognition rate with $20 \times 20$ feature size. It may be noted that the WFG-2DFLD method outperforms the 2DPCA [12], LDA [12], 2DFLD [12], G-2DFLD, and F-2DFLD [12] methods as demonstrated in Table I.

TABLE I: COMPARISON DIFFERENT METHODS IN TERMS OF RECOGNITION RATES (\%) ON THE YALE FACE DATABASE

\begin{tabular}{ccccccc}
\hline \multicolumn{7}{c}{ RATES $(\%)$ ON THE YALE FACE DATABASE } \\
& $\begin{array}{c}\text { 2DPCA } \\
{[12]}\end{array}$ & $\begin{array}{c}\text { LDA } \\
{[12]}\end{array}$ & $\begin{array}{c}\text { 2DFLD } \\
{[12]}\end{array}$ & $\begin{array}{c}\text { G-2D } \\
\text { FLD }\end{array}$ & $\begin{array}{c}\text { F-2DFLD } \\
{[12]}\end{array}$ & $\begin{array}{c}\text { WFG-2D } \\
\text { FLD } \\
(\beta=0.4)\end{array}$ \\
\hline $\begin{array}{c}\text { Result } \\
\begin{array}{c}\text { Feature } \\
\text { size }\end{array}\end{array}$ & 52 & 93.33 & 93.33 & 96 & 96 & $\mathbf{9 7 . 3 3}$ \\
& $50 \times 11$ & 14 & $50 \times 3$ & $18 \times 18$ & $50 \times 3$ & $20 \times 20$ \\
\hline
\end{tabular}

Highest recognition rates are indicated by the bold values.

\section{B. AT\&T Database}

This database consists of 400 images of 40 persons, where each person has 10 dissimilar images [28]. In our study, we have selected the $s$ images randomly from the pool of images from each person to generate the training set and remaining images were taken for creating test set. Therefore, the training and test set comprises completely distinct set of images. The values of $s$ are taken as 3, 4, 5, 6 and 7 to form different pairs of training and test sets. Fig. 3 represents the face images of two individuals.

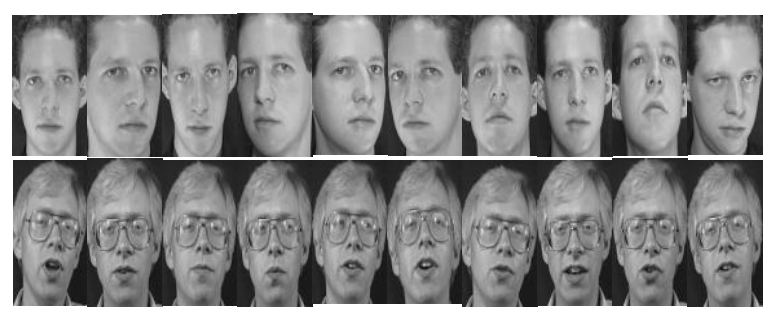

Fig. 3. Images of two different individuals in the AT\&T face database.

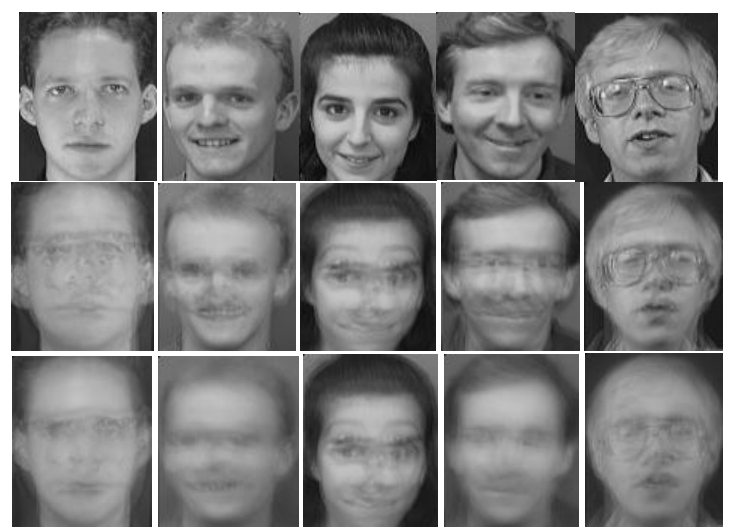

Fig. 4. Some example face images and corresponding class-wise mean images. Top row: example images from the AT\&T face database. Middle row: class-wise mean images. Bottom row: class-wise mean images using the WFG-2DFLD method.

In this study, we have validated the performance of our method with 20 different pairs of training and test sets for each value of $s$. Since the present method considers that a face image may simultaneously belong to different classes with possibly different membership values, the class-wise mean images may differ from the actual ones. In particular for $s=5$, Fig. 4 demonstrates that the WFG-2DFLD-based class-wise mean images (bottom row) actually differ from the actual ones (middle row).

For $s=5$, Fig. 5 shows the average recognition rates by varying the feature size and also the size of neighborhood $k$. From the figure it is clear that the suggested method provides superior result of $98 \%$ using $k=45$.

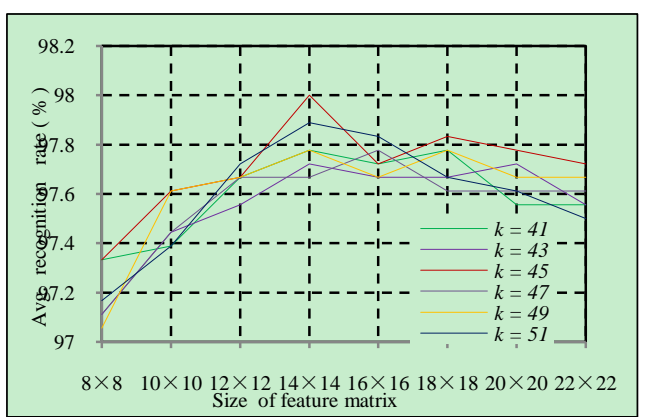

Fig. 5. Average recognition rates of the WFG-2DFLD method on the AT\&T database for different values $k$ and feature matrices.

In addition, Fig. 6 also shows the minimum, maximum and average recognition rates of the WFG-2DFLD method for different sizes of feature matrices using $k=45$.

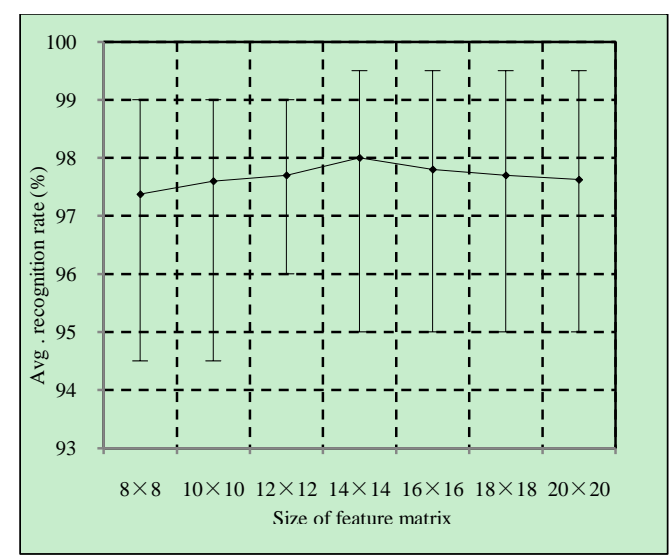

Fig. 6. Minimum, maximum and average recognition rates of the WFG-2DFLD method by varying feature matrices on the AT\&T face database using $k=45$.

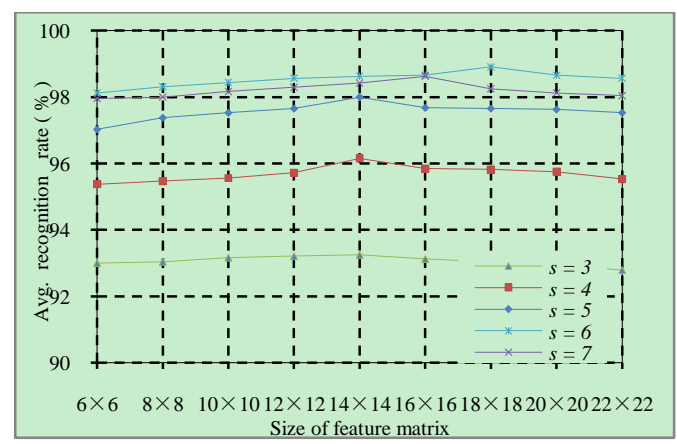

Fig. 7. Average recognition rates of the WFG-2DFLD method on the AT\&T database for different values of $s$ by varying the feature matrices.

Furthermore, the proposed method is also evaluated for $s=3,4,6$ and 7 by varying feature matrices. Fig. 7 shows the collective performance of the WFG-2DFLD method for different values of $s$. The proposed method yields the best average recognition rates of $93.25 \%(14 \times 14), 96.16 \%$ $(14 \times 14), 98 \%(14 \times 14), 98.91 \%(18 \times 18)$ and $98.82 \%(16 \times 16)$ for $s=3,4,5,6$ and 7 , respectively. 
The WFG-2DFLD method is compared with the MMSD [15], MSD [15], G-2DFLD [9], F-2DFLD [12], 2DFLD [9], Fuzzy fisherface [12], and 2DPCA [9] methods. The comparison in terms of best average recognition rates for different combination of training set and test set are presented in Table II. The results demonstrate that the WFG-2DFLD method outperforms the other methods. It may be noted that results of the Fuzzy fisherface [12] and F-2DFLD [12] methods are based on the 10 experimental runs.

TABLE II: COMPARISON IN TERMS OF AVERAGE RECOGNITION RATES OBTAINED FROM DiFFERENT METHODS ON THE AT\&T FACE DATABASE FOR DifFERENT VALUES OF $S$ AND Within THE PARENTHESIS THE FEATURE SizES ARE MENTIONED

\begin{tabular}{|c|c|c|c|c|c|}
\hline \multirow{2}{*}{ Method } & \multicolumn{5}{|c|}{ Average recognition rates } \\
\hline & $s=3$ & $s=4$ & $s=5$ & $s=6$ & $s=7$ \\
\hline \multirow{2}{*}{ WFG-2DFLD $(\beta=0.4)$} & 93.25 & 96.16 & 98.00 & 98.91 & 98.82 \\
\hline & $(14 \times 14)$ & $(14 \times 14)$ & $(14 \times 14)$ & $(18 \times 18)$ & $(16 \times 16)$ \\
\hline \multirow{2}{*}{$\operatorname{MMSD}(\beta=0.4)[15]$} & 90.23 & 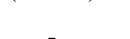 & 95.89 & 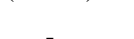 & 98.72 \\
\hline & (39) & - & (39) & - & $(39)$ \\
\hline \multirow{2}{*}{$\operatorname{MSD}(\beta=0.4)[15]$} & 89.15 & & 94.68 & & 98.51 \\
\hline & (39) & - & (39) & - & (39) \\
\hline \multirow{2}{*}{ G-2DFLD [9] } & 92.82 & 95.94 & 97.68 & 98.72 & 98.42 \\
\hline & $(16 \times 16)$ & $(16 \times 16)$ & $(14 \times 14)$ & $(14 \times 14)$ & $(8 \times 8)$ \\
\hline \multirow{2}{*}{ F-2DFLD [12] } & 92.08 & 95.04 & 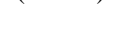 & ( & ( \\
\hline & $(56 \times 3)$ & $(56 \times 3)$ & - & - & - \\
\hline \multirow{2}{*}{ 2DFLD [9] } & 92.30 & 95.08 & 97.50 & 98.26 & 97.88 \\
\hline & $(112 \times 16)$ & $(112 \times 16)$ & $(112 \times 14)$ & $(112 \times 14)$ & $(112 \times 8)$ \\
\hline \multirow{2}{*}{ Fuzzy fisherface [12] } & 82.32 & 88.67 & _ & & - \\
\hline & $(39)$ & (39) & - & - & - \\
\hline \multirow{2}{*}{ 2DPCA [9] } & 91.27 & 94.33 & 96.83 & 97.72 & 97.79 \\
\hline & $(112 \times 16)$ & $(112 \times 16)$ & $(112 \times 14)$ & $(112 \times 14)$ & $(112 \times 8)$ \\
\hline
\end{tabular}

Highest recognition rates are indicated by the bold values.

\section{UMIST Database}

The UMIST face database is a multi-view database, which contains 575 grey scale images of 20 different persons (subjects) [29]. The images also cover a range of race, sex and appearance. The database does not contain fixed number of images per person; it varies from 19 images to 48 images per person. In the present study, each image of the database was cropped to $112 \times 92$ pixel. Fig. 8 shows some face images from the database.

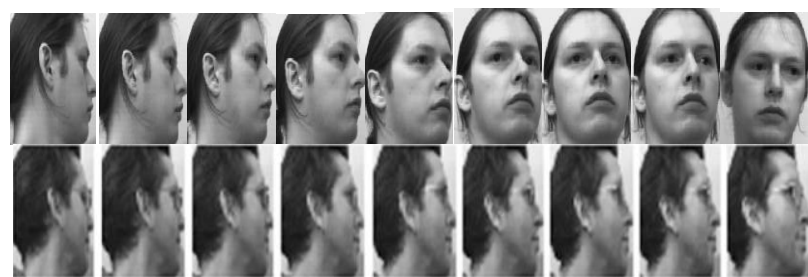

Fig. 8. Some face images of two different individuals from the UMIST database.

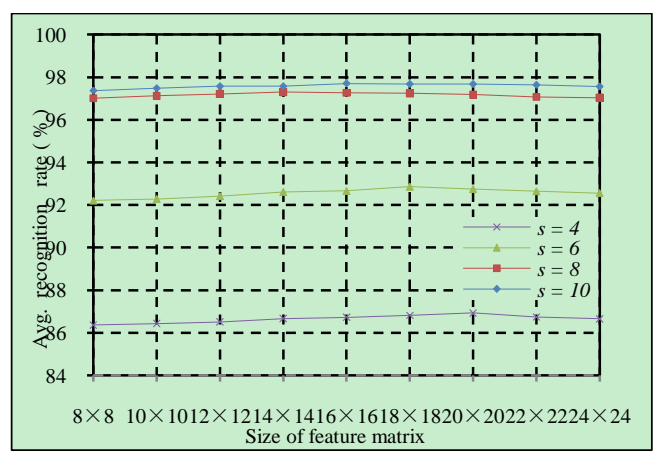

Fig. 9. Average recognition rates of the proposed WFG-2DFLD method on the UMIST database for different values of $s$ by varying the feature matrices.

In this experiment, like AT\&T database, we have selected the value of $s$ as 4, 6, 8 and 10 to form different pairs of training and test sets. It may be noted that there is no overlap between a pair of training and test sets in terms of face images. For each value of $s$, we have evaluated the performance of the proposed method with 20 different pairs of training and test sets. The proposed method is evaluated with feature matrices of sizes $8 \times 8,10 \times 10,12 \times 12,14 \times 14,16 \times 16,18 \times 18,20 \times 20$, $22 \times 22$ and $24 \times 24$ using RBFNN as a classifier. The RBFNN is modelled with 60, 100, 140 and 180 hidden layer nodes for 80, 120, 160 and 200 training images, respectively. Fig. 9 shows the recognition rates of the proposed method, which yields the best average recognition rates of $86.93 \%(20 \times 20)$, $92.86 \%(18 \times 18), 97.3 \%(14 \times 14)$ and $97.7 \%(16 \times 16)$ for $s=4$, 6,8 and 10 , respectively.

Table III shows a comparative presentation of the WFG-2DFLD method along with the G-2DFLD [9], 2DFLD [9], 2DPCA [9], PCA+FLD [9] and PCA [9] methods in terms of best average recognition rates. The results show that in all the cases, the performance of the WFG-2DFLD method is superior to the other methods.

TABLE III: COMPARISON IN TERMS OF AVERAGE RECOGNITION RATES (\%) OBTAINED FROM DIFFERENT METHODS ON THE UMIST FACE DATABASE. WiTHIN THE PARENTHESIS THE FEATURE SIZES ARE MENTIONED

\begin{tabular}{ccccc}
\hline \multirow{2}{*}{ Method } & \multicolumn{4}{c}{ Average recognition rates } \\
\cline { 2 - 5 } & $s=4$ & $s=6$ & $s=8$ & $s=10$ \\
\hline WFG-2DFLD & $\mathbf{8 6 . 9 3}$ & $\mathbf{9 2 . 8 6}$ & $\mathbf{9 7 . 3 0}$ & $\mathbf{9 7 . 7 0}$ \\
$(\beta=0.4)$ & $(20 \times 20)$ & $(18 \times 18)$ & $(14 \times 14)$ & $(16 \times 16)$ \\
G-2DFLD [9] & 86.22 & 92.28 & 95.54 & 96.92 \\
& $(14 \times 14)$ & $(14 \times 14)$ & $(14 \times 14)$ & $(18 \times 18)$ \\
2DFLD [9] & 86.12 & 92.16 & 95.25 & 96.55 \\
& $(112 \times 14)$ & $(112 \times 14)$ & $(112 \times 14)$ & $(112 \times 18)$ \\
2DPCA [9] & 85.70 & 91.91 & 95.07 & 96.60 \\
& $(112 \times 14)$ & $(112 \times 14)$ & $(112 \times 14)$ & $(112 \times 18)$ \\
PCA+FLD [9] & 76.31 & 85.69 & 90.93 & 93.72 \\
& $(25)$ & $(25)$ & $(25)$ & $(25)$ \\
PCA [9] & 80.72 & 86.53 & 94.01 & 95.11 \\
& $(60)$ & $(60)$ & $(60)$ & $(60)$ \\
\hline
\end{tabular}

Highest recognition rates are indicated by the bold values.

\section{FERET Database}

The FERET face database was formed through the FERET program. It was sponsored by the US Department of Defence through the DARPA program [30, 31]. The proposed method was performed on a subset of this database which comprises 1400 images of 200 individuals, each individual having 7 
images. The images are of different variations in facial expression, illumination and pose. In our experiment, the facial portion of each original image was manually cropped and resized to $80 \times 80$ pixels based on the location of the eyes. Some examples images of two persons are shown in Fig. 10.

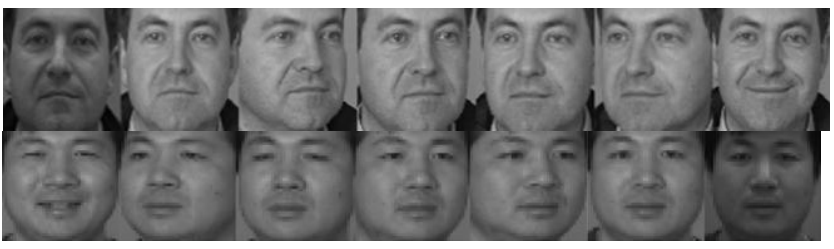

Fig. 10. Sample images of two different individuals from the FERET database.

To reduce the influence of performance of training and test set, for each value of $s$, experiments are repeated 10 times with different training and test sets. Here, we choose $s=2,3$, 4 images from each subject at random for training and remaining (7-s) images are employed for testing. The proposed method is evaluated with feature matrices of sizes $6 \times 6,8 \times 8,10 \times 10,12 \times 12,14 \times 14,16 \times 16,18 \times 18$ and $20 \times 20$ using RBFNN as a classifier. The RBFNN is modeled with 400, 400 and 600 hidden layer nodes for 400, 600 and 800 training images, respectively. Fig. 11 shows the recognition rates of the proposed method, which yields the best average recognition rates of $49.46 \%(6 \times 6), 59.41 \%(12 \times 12)$ and $65.88 \%(12 \times 12)$ for $s=2,3$ and 4 , respectively.

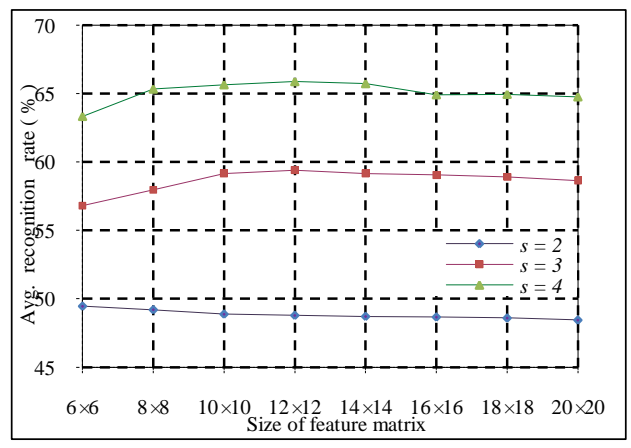

Fig. 11. Average recognition rates of the proposed WFG-2DFLD method on the FERET database for different values of $s$ by varying the size of feature matrices.

TABLE IV: COMPARISON IN TERMS OF AVERAGE RECOGNITION RATES (\%) OBTAINED FROM DIFFERENT METHODS ON THE FERET FACE DATABASE. WITHIN THE PARENTHESIS THE FEATURE SiZES ARE MENTIONED

\begin{tabular}{cccc}
\multicolumn{2}{c}{ WITHIN THE PARENTHESIS THE FEATURE SIZES ARE MENTIONED } \\
\cline { 2 - 4 } Method & \multicolumn{3}{c}{ Average recognition rates } \\
\cline { 2 - 4 } WFG-2DFLD & $\mathbf{4 9 . 4 6}$ & $\mathbf{5 9 . 4 1}$ & $s=4$ \\
$(\beta=0.4)$ & $(6 \times 6)$ & $(12 \times 12)$ & $\mathbf{6 5 . 8 8}$ \\
FMSD $(\beta=0.4)$ & - & 53.46 & 56.9 \\
{$[15]$} & & $(40)$ & $(40)$ \\
MMSD $(\beta=0.4)$ & - & 52.6 & 55.81 \\
{$[15]$} & & $(40)$ & $(40)$ \\
MSD $(\beta=0.4)$ & - & 50.5 & 53.68 \\
{$[15]$} & 48.31 & $(40)$ & $(40)$ \\
Alternative-2DPCA & 53.21 & 53.97 \\
{$[26]$} & $(112 \times 20)$ & $(112 \times 20)$ & $(112 \times 20)$ \\
(2D) 2PCA [26] & $(11.70$ & 52.36 & 55.45 \\
& 47.12 & $(112 \times 20)$ & $(112 \times 20)$ \\
2DPCA [26] & $(112 \times 20)$ & $(112 \times 20)$ & $(112 \times 20)$ \\
\hline
\end{tabular}

Highest recognition rates are indicated by the bold values.

We have compared the performance of the proposed WFG-2DFLD method with the FMSD [15], MMSD [15], MSD [15], Alternative-2DPCA [26], (2D) 2PCA [26] and 2DPCA [26] methods. Table IV illustrates that our proposed WFG-2DFLD method has the highest average recognition rates of $49.46 \%(6 \times 6), 59.41 \%(12 \times 12)$ and $65.88 \%(12 \times 12)$ using 2, 3 and 4 training samples per class, respectively. Thus, the results again demonstrate the superiority of the WFG-2DFLD method over other methods.

\section{CONCLUSION}

In this paper, we have proposed a new feature extraction algorithm namely, weighted fuzzy generalized two-dimensional Fisher's linear discriminant (WFG-2DFLD) method for face recognition. The method assumes that a face image may belong to several classes with possibly different membership values. These membership values are generated by fuzzy $k$-NN algorithm and used to generate fuzzy global mean image and fuzzy class-wise mean images. Finally these mean images are used to generate fuzzy within-class and fuzzy between-class scatter matrices along row and column directions. In order to make more accurate for classification, weights $(\beta=0.4)$ are assigned to fuzzy within-class and fuzzy between-class scatter matrices along row and column directions. The projection matrices obtained by solving these scatter matrices, satisfying the two Fisher's criteria, yield rich information leading to generation of superior discriminant features. Image classification and recognition is performed using a RBF neural network. Its efficiency is demonstrated by the superior results on the YALE, AT\&T, UMIST and FERET face databases. The empirical results suggest that the proposed WFG-2DFLD method is more superior for the task of face recognition.

\section{ACKNOWLEDGMENT}

This work was supported by the Senior Research Fellowship Program of Aniruddha Dey under the State Government Fellowship (Ref. No. - P-1/RS./365/12, dated $05^{\text {th }}$ October, 2012.) of the Department of Computer Science \& Engineering, Jadavpur University, Kolkata. The authors would also like to thank Mr. Sayan Kahali, Department of Computer Science \& Engineering, Jadavpur University for several discussions which improve the presentation of the paper considerably.

\section{REFERENCES}

[1] H. Zhou, A. Mian, L. Wei, D. Creighton, M. Hossny, and S. Nahavandi, "Recent advances on singlemodal and multimodal face recognition: A survey," IEEE Trans. Human Machine Systems, vol. 44, no. 6, pp. 701-716, Dec. 2014.

[2] B. Poon, M. A. Amin, and H. Yan, "Performance evaluation and comparison of PCA based human face recognition methods for distorted images," International Journal of Machine Learning and Cybernetics, vol. 2, no. 4, pp. 245-259, July 2011.

[3] G. J. Alvarado, W. Pedrycz, M. Reformat, and K.-C. Kwak, "Deterioration of visual information in face classification using eigenfaces and fisherfaces," Machine Vision and Applications, vol. 17, no. 1, pp. 68-82, March 2006.

[4] X. S. Zhuang and D. Q. Dai, "Improved discriminant analysis for high-dimensional data and its application to face recognition," Pattern Recogn., vol. 40, no. 5, pp. 1570-1578, May 2007.

[5] M. J. Er, S. Wu, J. Lu, and H. L. Toh, "Face recognition with radial basis function (RBF) neural networks," IEEE Trans. Neural Netw., vol. 13, no 3, pp. 697-710, August 2002.

[6] M. S. Bartlet, J. R. Movellan, and T. J. Sejnowski, "Face recognition by independent component analysis," IEEE Trans. Neural Netw., vol. 13, no 6, pp. 1450-1464, November 2002.

[7] J. Yang, D. Zhang, A. F. Frangi, and J. Y. Yang, "Two-dimensional PCA: A new approach to appearance-based face representation and 
recognition," IEEE Trans. Pattern Anal. Mach. Intell., vol. 26, no. 1, pp. 131-137, June 2004

[8] H. Xiong, M. N. S. Swamy, and M. O. Ahmad, "Two-dimensional FLD for face recognition," Pattern Recogn., vol. 38, no. 7, pp. 1121-1124, July 2005.

[9] S. Chowdhury, J. K. Sing, D. K. Basu, and M. Nasipuri, "Face recognition by generalized two-dimensional FLD method and multi-class support vector machines," Appl. Soft Comput., vol. 11, no.7, pp. 4282-4292, Oct. 2011

[10] J. M. Keller, M. R. Gray, and J. A. Givens, “A fuzzy $k$-nearest neighbor algorithm," IEEE Trans. Syst. Man Cybernet., vol. 15, no. 4, pp. 580-585, August 1985

[11] K. C. Kwak and W. Pedrycz, "Face recognition using a fuzzy fisherface classifier," Journal of the Pattern Recogn, vol. 38, no. 10, pp 1717-1732, Oct. 2005.

[12] W. Yang, J. Wang, M. Ren, and J. Yang, "Fuzzy 2-dimensional FLD for face recognition," Journal of Information and Computing Science, vol. 4, no. 3, pp. 233-239, 2009.

[13] W. Yang, J. Wang, M. Ren, L. Zhang, and J. Yang, "Feature extraction using fuzzy inverse FDA," Neurocomputing, vol. 72, no. 13-15, pp. 3384-3390, August 2009.

[14] X. Li, "Face recognition method based on fuzzy 2DPCA," Journal of Electrical and Computer Engineering, pp. 1- 7, May 2014.

[15] X. Li and A. Song, "Fuzzy MSD based feature extraction method for face recognition," Neurocomputing, vol. 112, no. 13-15, pp. 266-271, December 2013.

[16] J. K. Sing, D. K. Basu, M. Nasipuri, and M. Kundu, "Face recognition using point symmetry distance-based RBF network," Appl. Soft Comput., vol. 7, no. 1, pp. 58-70, January 2007.

[17] J. K. Sing, S. Thakur, D. K. Basu, M. Nasipuri, and M. Kundu, "High-speed face recognition using self-adaptive radial basis function neural networks," Neural Comput. Appl., vol. 18, no. 8, pp. 979-990, November 2009.

[18] G. Heusch, and S. Marcel, "A novel statistical generative model dedicated to face recognition," Image Vis. Comput., vol. 28, no. 1, pp 101-110, January 2010.

[19] J. K. Sing, S. Chowdhury, D. K. Basu, and M. Nasipuri, "An improved hybrid approach to face recognition by fusing local and globa discriminant feature," Int. J. Biometrics, vol. 4, no. 2, pp. 144-164 2012.

[20] O. Rasanen and U. K. Laine, "A method for noise-robust context-aware pattern discovery and recognition from categorical sequences," Pattern Recogn., vol. 45, no.1, pp. 606-616, January 2012

[21] M. Hanmandlu, D. Gupta, and S. Vasikarla, "Face recognition by elastic bunch graph matching," in Proc. the IEEE Conference on Applied Imagery Pattern Recognition Workshop (AIPR): Sensing for Control and Augmentation, 2013, pp. 1-7.

[22] A. Pentland, B. Moghaddam, and T. Starner, "View-based and modular eigenspaces for face recognition," in Proc. the IEEE Conference on Computer Vision and Pattern Recognition, 1994, pp. 1-7.

[23] E. Fazl-Ersi, and J. K. Tsotsos, "Local feature analysis for robust face recognition," in Proc. the IEEE Symposium on Computational Intelligence for Security and Defense Applications, 2009, pp. 1-6.

[24] S. Du and K. R. Ward, "Adaptive region-based image enhancement method for robust face recognition under variable illumination conditions," IEEE Trans. on Circuits and Systems for Video Tech., vol. 20, no. 9, pp. 1165-1175, Sep. 2010.

[25] B. Weyrauch, J. Huang, B. Heisele, and V. Blanz, "Component-based face recognition with 3D morphable models," in Proc. the International Conference on Computer Vision and Pattern Recognition Workshops, July 2004.

[26] Q. Zhu and Y. Xu, "Multi-directional two dimensional PCA with matching score level fusion for face recognition," Neural Comput. \& Applic., vol. 23, no. 1, pp. 169-174, July 2013.
[27] The Yale face database. [Online]. Available: http://cvc.yale.edu/projects/yalefaces/yalefaces.html

[28] The ORL face database. [Online]. Available: http://www.cl.cam.ac.uk/research/dtg/attarchive/facedatabase.html

[29] D. B. Graham, N. M. Allinson et al., "Characterizing virtual eigen signatures for general purpose face recognition: From theory to applications," NATO ASI Series F Computer and Systems Sciences, vol. 163, pp. 446-456, 1998.

[30] P. J. Phillips, H. Moon, S. A. Rizvi, and P. J. Rauss, "The FERET evaluation methodology for face-recognition algorithms," IEEE Trans. Pattern. Anal. Mach. Intell., vol. 22, pp. 1090-1104, 2000.

[31] P. J. Phillips, The Facial Recognition Technology (FERET) Database, 2004.

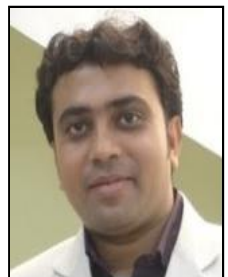

Aniruddha Dey received his B.Tech degree in computer science and engineering from West Bengal University of Technology (now Maulana Abul Kalam Azad University of Technology), Kolkata, India, in 2007, and M.Tech.IT degree in courseware engineering from Jadavpur University, Kolkata, India, in 2010 . He is presently a senior research fellow (under state government fellowship) at the Department of Computer Science \& Engineering, Jadavpur University, and Kolkata, India since October 2012. He is currently pursuing his doctorate of philosophy in computer science and engineering at Jadavpur University. His research interests include face recognition and detection, pattern recognition and image processing.

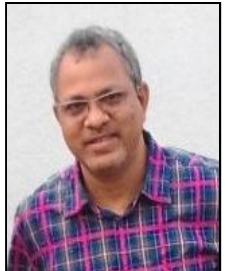

Jamuna Kanta Sing has received his B.E. degree in computer science \& engineering from Jadavpur University in 1992, M.Tech. degree in computer \& information technology from Indian Institute of Technology (IIT) Kharagpur in 1994 and Ph.D. (engineering) degree from Jadavpur University in 2006. Dr. Sing has joined the Department of Computer Science \& Engineering, Jadavpur University in March 1997 and presently serving as a professor since 2010. He is a recipient of the BOYSCAST fellow of the Department of Science \& Technology, Govt. of India for doing advanced research at the University of Pennsylvania and the University of Iowa, USA in 2006 and the UGC Research Award in 2014. He is a senior member of the IEEE, USA. He has published more than 35 research papers in reputed refereed International Journals and more than 60 papers in international conferences. He has supervised $8 \mathrm{PhD}$ scholars and handled (including on-going) $5 \mathrm{R} \& \mathrm{D}$ projects from the AICTE, UGC and DST of worth around Rs. 65 Lakhs. His research interest includes face recognition and detection, video analytics, medical image processing, and pattern recognition.

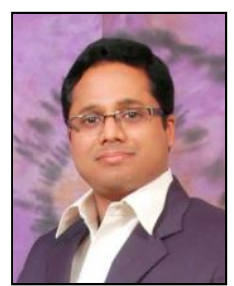

Shiladitya Chowdhury received his bachelor of technology degree in computer science and engineering from West Bengal University of Technology (now Maulana Abul Kalam Azad University of Technology), India, in 2005. He received his master of technology degree in computer technology and Ph.D. degree in engineering from Jadavpur University, India, in 2009 and 2016, respectively. Dr. Chowdhury has been a faculty member of the Department of Master of Computer Application, Techno India, Saltlake, India, since 2007. His current fields of research interest include face recognition, pattern recognition and image processing. 